\title{
Correlation between human epidermal growth factor receptor 2 expression level and efficacy of trastuzumab beyond progression in metastatic gastric cancer
}

\author{
YUKIYA NARITA $^{1 *}$, SHIGENORI KADOWAKI $^{1 *}$, TOSHIKI MASUISHI $^{1}$, HIROYA TANIGUCHI $^{1}$, \\ DAISUKE TAKAHARI ${ }^{1}$, TAKASHI URA ${ }^{1}$, MASASHI ANDO ${ }^{1}$, MASAHIRO TAJIKA ${ }^{2}$, \\ YASUMASA NIWA ${ }^{2}$, TETSUYA ETO ${ }^{3}$, HIROKI HARA ${ }^{4}$, MASAKO ASAYAMA ${ }^{4}$, \\ KENSEI YAMAGUCHI ${ }^{4}$, YASUSHI YATABE ${ }^{5}$ and KEI MURO ${ }^{1}$
}

\begin{abstract}
Departments of ${ }^{1}$ Clinical Oncology and ${ }^{2}$ Endoscopy, Aichi Cancer Center Hospital, Nagoya, Aichi 464-8681;
${ }^{3}$ Department of Gastroenterology, Tsuchiura Kyodo General Hospital, Tsuchiura, Ibaraki 300-0028; ${ }^{4}$ Department of Gastroenterology, Saitama Cancer Center Hospital, Saitama 362-0806; ${ }^{5}$ Department of Pathology and Molecular Diagnostics, Aichi Cancer Center Hospital, Nagoya, Aichi 464-8681, Japan
\end{abstract}

Received February 4, 2016; Accepted January 6, 2017

DOI: $10.3892 / 01.2017 .6409$

\begin{abstract}
There is currently no clinical data regarding the efficacy of trastuzumab treatment for the progression of human epidermal growth factor receptor 2 (HER2)-positive advanced gastric cancer (AGC) occurring during trastuzumab-based chemotherapy. The aim of this study was to retrospectively examine the clinical benefits of trastuzumab for HER2-positive AGC patients who progressed during first-line trastuzumab-based chemotherapy. Among the 108 patients treated with trastuzumab combined with fluoropyrimidine and cisplatin as first-line therapy, 46 HER2-positive AGC patients who received cytotoxic agents with or without trastuzumab subsequent to disease progression were included. Of these,
\end{abstract}

Correspondence to: Dr Shigenori Kadowaki, Department of Clinical Oncology, Aichi Cancer Center Hospital, 1-1 Kanokoden, Chikusa-ku, Nagoya, Aichi 464-8681, Japan

E-mail: skadowaki@aichi-cc.jp

*Contributed equally

Abbreviations: AGC, advanced gastric cancer; CI, confidence intervals; CR, complete response; DCR, disease control rate; FISH, fluorescent in situ hybridization; HER2, human epidermal growth factor receptor 2; HR, hazard ratio; IHC, immunohistochemistry; non-TBP, discontinued trastuzumab beyond progression; ORR, objective response rate; OS, overall survival; PFS, progression-free survival; PR, partial response; $\mathrm{SD}$, stable disease; TBP, trastuzumab beyond progression; ToGA trial, Trastuzumab for Gastric Cancer trial; TyTAN trial, Tykerb with Taxol in Asian ErbB2+ Gastric Cancer trial

Key words: gastric cancer, human epidermal growth factor receptor 2, metastatic gastric cancer, trastuzumab, trastuzumab beyond progression the efficacy and safety outcomes of 26 patients who continued trastuzumab were compared with those of the 20 patients who discontinued trastuzumab. No difference in response rate (18.2 vs. $15.8 \%, \mathrm{P}=1.00$ ) was observed between the two groups. Progression-free survival (PFS) time was numerically longer in the chemotherapy combination with trastuzumab group than in the chemotherapy combination without trastuzumab group (median, 4.0 vs. 2.3 months), with no significance [hazard ratio (HR), $0.63 ; \mathrm{P}=0.14]$. In the subset analysis, continuation of trastuzumab significantly improved PFS time in selected subgroups of patients with tumors exhibiting HER2 expression scores of $3+(\mathrm{HR}, 0.41 ; \mathrm{P}=0.04)$, intestinal-type histology (HR, 0.32; $\mathrm{P}<0.01)$, and a first PFS time of $>6$ months $(\mathrm{HR}$, $0.44 ; \mathrm{P}=0.04)$. The survival times for the trastuzumab beyond progression (TBP) and non-TBP groups were similar (HR, 1.06; $\mathrm{P}=0.88$ ), with equivalent overall survival times in the subgroups with immunohistochemistry scores of $3+(\mathrm{HR}, 0.97 ; \mathrm{P}=0.94)$, intestinal-type histology $(\mathrm{HR}, 0.53 ; \mathrm{P}=0.19)$, and a first $\mathrm{PFS}$ time of $>6$ months (HR, $0.62 ; \mathrm{P}=0.31$ ). There were no differences in the incidence rates of toxicity, including cardiac dysfunction, between the two groups. The study results suggest that selected HER2-positive AGC patients may benefit from trastuzumab continuation during first progression, and further prospective studies are warranted.

\section{Introduction}

Gastric cancer is the third leading cause of cancer-associated mortality, with an estimated 723,027 mortalities worldwide in 2012 (1). Compared with the best supportive care, first-line chemotherapy for advanced gastric cancer (AGC) improves survival time and quality of life (2-4); however, the median overall survival (OS) time is short (9-13 months) (5-7). Several previous studies have demonstrated that second-line chemotherapy confers a significant survival benefit over best supportive care in AGC, with median OS time ranging from 4 to 9.5 months (8-11). 
Among patients with AGC, the rate of human epidermal growth factor receptor 2 (HER2) positivity reportedly ranges from 8.2 to $23.0 \%$ as assessed by immunohistochemistry (IHC), and from 7.1 to $27.1 \%$ when assessed by fluorescence in situ hybridization (FISH) (12-15). On the basis of the Trastuzumab for Gastric Cancer (ToGA) trial results, addition of trastuzumab to platinum-based chemotherapy has become the standard first-line therapy for HER2-positive AGC (16). However, in the second-line setting, the Tykerb with Taxol in Asian ErbB2+ Gastric Cancer (TyTAN) trial did not demonstrate a survival benefit from the addition of lapatinib, a small-molecule tyrosine kinase inhibitor targeting HER2, to paclitaxel for the treatment of HER2-positive AGC (17). Preliminary evidence from a small phase II study demonstrated that a trastuzumab plus docetaxel-containing regimen was well tolerated and effective for HER2-positive AGC previously treated with fluorouracil-based chemotherapy; however, in this study, the patients had received no previous therapy with trastuzumab (18). On the other hand, several studies have reported that continued therapy with trastuzumab beyond progression (TBP) improves the survival outcome of patients with metastatic HER2-positive breast cancer $(19,20)$, and represents a practical therapeutic option. However, there is currently no clinical data regarding the efficacy of trastuzumab for HER2-positive AGC progressing during trastuzumab-based chemotherapy. The aim of the present, retrospective study was to assess the clinical benefit of the continuation of trastuzumab for HER2-positive AGC patients who progressed during trastuzumab-based chemotherapy.

\section{Patients and methods}

Patients. The study population comprised patients with HER2-positive AGC who were treated at three Japanese institutions (Aichi Cancer Center Hospital, Nagoya, Japan; Tsuchiura Kyodo General Hospital, Tsuchiura, Japan; and Saitama Cancer Center Hospital, Saitama, Japan) between January 2006 and March 2014. The principal inclusion criteria were as follows: Histologically proven inoperable gastric cancer; an Eastern Cooperative Oncology Group performance status of 0-2 (21); sufficient bone marrow, liver and renal function; progressive disease diagnosed by computed tomography scans during first-line chemotherapy of trastuzumab combined with fluoropyrimidine plus platinum; and treatment with cytotoxic agents (docetaxel, $60 \mathrm{mg} / \mathrm{m}^{2}$ every 3 weeks; paclitaxel $80 \mathrm{mg} / \mathrm{m}^{2}$ on days 1,8 and 15 , every 4 weeks; irinotecan $150 \mathrm{mg} / \mathrm{m}^{2}$ every 2 weeks; or irinotecan $60 \mathrm{mg} / \mathrm{m}^{2}$ plus cisplatin $30 \mathrm{mg} / \mathrm{m}^{2}$ every 2 weeks) with or without trastuzumab $(8 \mathrm{mg} / \mathrm{kg}$ on day 1 of the first cycle, followed by $6 \mathrm{mg} / \mathrm{kg}$ every 3 weeks) as second-line chemotherapy. The second-line treatment regimens were selected by the treating physicians at each institution. HER2-positivity was defined as an IHC score of 3+, or an IHC score of 2+ and an in situ hybridization (ISH)-positive result determined by FISH or dual-color ISH $(16,22)$, which are considered to be indications for using trastuzumab on the basis of subset analysis of the ToGA trial results $(16,22)$. The HER2 status examination was performed prior to the start of the first-line chemotherapy. Written informed consent for treatment was obtained from each patient prior to treatment initiation. The Institutional Review Board of each participating center approved the study.
Data analysis. The main purpose of this study was to compare the efficacy and safety of trastuzumab treatment for patients who subsequently continued trastuzumab (TBP group) vs. those who discontinued trastuzumab beyond progression (non-TBP group). Treatment responses were evaluated in accordance with the Response Evaluation Criteria in Solid Tumors (version 1.1) (23) and the best overall response was recorded as the antitumor effect for each patient. The objective response rate (ORR) was defined as the proportion of patients with a complete response (CR) or a partial response (PR), and the disease control rate (DCR) was defined as the proportion of patients with a CR, a PR or stable disease (SD).

Progression-free survival (PFS) time was measured from the date of second-line therapy initiation to the date of progressive disease or mortality due to any cause. OS time was calculated from the date of second-line therapy initiation to the date of mortality or the last follow-up visit. The PFS and OS curves were constructed by using the Kaplan-Meier method. Survival status was updated in October 2014.

The P-values for the comparison of differences between the baseline patient characteristics of each group were calculated using $\chi^{2}$ tests for homogeneity or trend, or by using Fisher's exact test. Univariate Cox proportional hazards models were used to assess the effects of TBP in terms of PFS and OS, which were indicated by hazard ratios (HR) and $95 \%$ confidence intervals (CI). The incidence and severity of adverse events were graded using the National Cancer Institute Common Toxicity Criteria version 4.0 (24). Statistical analyses were performed with $\mathrm{R}$ software version 2.13.2 (R Project for Statistical Computing, Vienna, Austria). All tests were two-tailed and $\mathrm{P}<0.05$ was considered to indicate statistical significance.

\section{Results}

Patient characteristics. The characteristics of the patients are summarized in Table I. During the study period, 108 consecutive patients with HER2-positive AGC received trastuzumab combined with fluoropyrimidine plus platinum as first-line treatment, of which 34 patients enrolled in clinical trials, 28 patients did not receive second-line chemotherapy, and 46 patients met the inclusion criteria. A total of 26 patients (57\%) continued and $20(43 \%)$ did not continue trastuzumab. For all patients, HER2 status could not be tested at the time of disease progression during trastuzumab treatment. Between the TBP and non-TBP groups, no statistically significant differences were identified in histological subtypes (intestinal-type, 61.5 vs. $65.0 \%$, respectively; $\mathrm{P}=0.81$ ), IHC score $(3+, 80.8$ vs. $60.0 \%$, respectively; $\mathrm{P}=0.19)$, presence of one or more measurable lesions (84.6 vs. $95.0 \%$, respectively; $\mathrm{P}=0.34$ ), time-to-first disease progression (first PFS time, 6.4 vs. 6.2 months respectively; log-rank $\mathrm{P}=0.59$ ), or $>6$-month first PFS rate (57.7 vs. $60.0 \%$, respectively; $\mathrm{P}=0.87$ ). In the TBP group, for second-line chemotherapy, 22 patients (84.6\%) received trastuzumab plus taxanes, 3 patients (11.6\%) received trastuzumab plus irinotecan, and 1 (3.8\%) received cisplatin plus irinotecan combined with trastuzumab. For third-line chemotherapy, 7 patients (26.9\%) received taxanes/irinotecan and $8(30.8 \%)$ received taxanes/irinotecan combined with trastuzumab. In the non-TBP group, 13 patients $(65.0 \%)$ received taxanes, and the remaining patients $(35.0 \%)$ received 
Table I. Patient characteristics.

\begin{tabular}{|c|c|c|c|}
\hline Characteristic & Non-TBP group $(n=20)$ & TBP group $(n=26)$ & P-value \\
\hline Age, years [median (range)] & $64(33-74)$ & $62(29-86)$ & $0.97^{\mathrm{b}}$ \\
\hline Gender, n (\%) & & & $0.40^{\mathrm{c}}$ \\
\hline Male & $14(70.0)$ & $21(80.8)$ & \\
\hline Female & $6(30.0)$ & $5(19.2)$ & \\
\hline ECOG PS, n (\%) & & & $0.26^{\mathrm{c}}$ \\
\hline 0 & $9(45.0)$ & $6(23.1)$ & \\
\hline 1 & $9(45.0)$ & $18(69.2)$ & \\
\hline 2 & $2(10.0)$ & $2(7.7)$ & \\
\hline Disease status, n (\%) & & & $0.71^{\mathrm{c}}$ \\
\hline Unresectable & $17(85.0)$ & $20(76.9)$ & \\
\hline Recurrent & $3(15.0)$ & $6(23.1)$ & \\
\hline Number of metastatic sites, n (\%) & & & $0.73^{c}$ \\
\hline 1 & $4(20.0)$ & $7(26.9)$ & \\
\hline$\geq 2$ & $16(80.0)$ & $19(73.1)$ & \\
\hline \multicolumn{4}{|l|}{ Metastatic sites, n (\%) } \\
\hline Liver & $12(60.0)$ & $11(42.3)$ & $0.23^{\mathrm{b}}$ \\
\hline Lung & $3(15.0)$ & $9(34.6)$ & $0.18^{c}$ \\
\hline Lymph node & $15(75.0)$ & $22(84.6)$ & $0.47^{c}$ \\
\hline Peritoneum & $12(60.0)$ & $10(38.5)$ & $0.14^{\mathrm{b}}$ \\
\hline Prior gastrectomy, n (\%) & & & $0.34^{\mathrm{c}}$ \\
\hline Yes & $4(20.0)$ & $9(34.6)$ & \\
\hline Histological subtypes, n (\%) & & & $0.81^{\mathrm{b}}$ \\
\hline Diffuse & $7(35.0)$ & $10(38.5)$ & \\
\hline Intestinal & $13(65.0)$ & $16(61.5)$ & \\
\hline HER2 status, n (\%) & & & $0.19^{c}$ \\
\hline IHC 2+/FISH positive & $8(40.0)$ & $5(19.2)$ & \\
\hline IHC 3+ & $12(60.0)$ & $21(80.8)$ & \\
\hline$\geq 1$ measureable target lesion, $\mathrm{n}(\%)$ & & & $0.34^{\mathrm{c}}$ \\
\hline Yes & $19(95.0)$ & $22(84.6)$ & \\
\hline Adjuvant chemotherapy, n (\%) & & & $1.00^{\mathrm{c}}$ \\
\hline Yes & $2(10.0)$ & $3(11.5)$ & \\
\hline Prior chemotherapy, n (\%) & & & $0.01^{\mathrm{c}}$ \\
\hline $\mathrm{XP}$ with trastuzumab & $19(95.0)$ & $19(73.1)$ & \\
\hline SP with trastuzumab & $0(0)$ & $7(26.9)$ & \\
\hline FP with trastuzumab & $1(5.0)$ & $0(0)$ & \\
\hline Second-line chemotherapy, n (\%) & & & $0.16^{\mathrm{d}, \mathrm{c}}$ \\
\hline Taxanes $^{\mathrm{a}}$ & $13(65.0)$ & $22(84.6)$ & \\
\hline Irinotecan & $7(35.0)$ & $4(15.4)$ & \\
\hline Cisplatin & $0(0)$ & $1(3.8)$ & \\
\hline Trastuzumab & $0(0)$ & $26(100)$ & \\
\hline PFS of first-line chemotherapy, months [median (range)] & $6.2(2.118 .1)$ & $6.4(1.621 .6)$ & $0.59^{\mathrm{e}}$ \\
\hline
\end{tabular}

${ }^{a}$ Paclitaxel or docetaxel; ${ }^{\mathrm{b}} \chi^{2}$ tests; ${ }^{\mathrm{c}}$ Fisher's exact test; ${ }^{\mathrm{d}}$ comparison of taxanes, irinotecan and cisplatin, excluding trastuzumab; ${ }^{\mathrm{e}}$ log-rank test. TBP, trastuzumab beyond progression; ECOG, Eastern Cooperative Oncology Group; PS, performance status; HER2, human epidermal growth factor receptor 2; IHC, immunohistochemistry; FISH, fluorescence in situ hybridization; PFS, progression-free survival; XP, capecitabine plus cisplatin; SP, S-1 plus cisplatin; FP, 5-FU plus cisplatin.

irinotecan as second-line therapy. For third-line chemotherapy, 12 patients $(60.0 \%)$ received taxanes/irinotecan and
$3(15.0 \%)$ received taxanes/irinotecan combined with trastuzumab. 

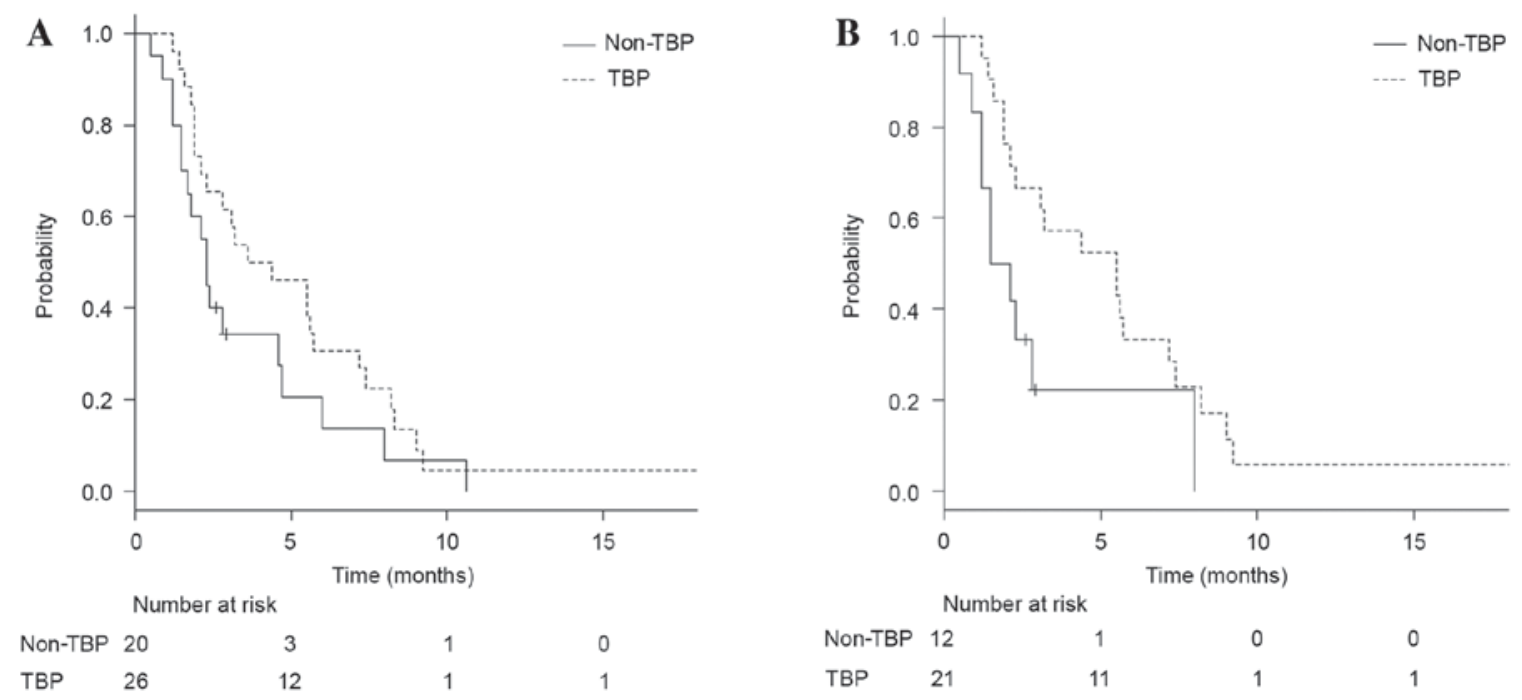

Figure 1. Kaplan-Meier survival curves of progression-free survival time for (A) all patients and (B) patients with an immunohistochemistry score of 3+ for epidermal growth factor receptor 2. TBP, trastuzumab beyond progression.

Treatment and efficacy. At a median follow-up time of 8.7 months, $43(87.8 \%)$ patients had experienced disease progression and $32(70 \%)$ patients had succumbed to the disease at the time of analysis. In the TBP group, among the 22 assessable patients, no patients achieved CR, 3 achieved PR, and 9 achieved SD, with an ORR of $13.6 \%$ and a DCR of $54.5 \%$. In the non-TBP group, among the 19 assessable patients, 3 achieved PR, and 4 achieved SD, with an ORR of $15.8 \%$ and a DCR of $36.8 \%$. The differences between the two groups were not significant, with P-values of 1.00 and 0.21 for ORR and DCR, respectively. No significant differences in response rates for any subgroups (age, gender, ECOG PS, disease status, number of metastatic sites, metastatic sites, prior gastrectomy, histological subtypes, HER2 status, adjuvant chemotherapy) between the TBP group and non-TBP group were identified.

Only 3 patients had not experienced disease progression at the time of analysis. Addition of trastuzumab (vs. no addition) numerically improved PFS time, with no significance (median, 4.0 vs. 2.3 months, respectively; HR, 0.63; 95\% CI, 0.34-1.17; $\mathrm{P}=0.14$; Fig. 1A). In the exploratory subset analysis of PFS time, three subgroups that could potentially benefit from continuing TBP vs. not continuing TBP were identified: Patients with a HER2 IHC score of $3+$ (median, 5.5 vs. 1.8 months, respectively; HR, 0.41; 95\% CI, 0.18-0.94; P=0.04; Fig. 1B), patients with histology of intestinal type (median, 5.7 vs. 2.3 months, respectively; HR, 0.32; 95\% CI, 0.14-0.75; $\mathrm{P}<0.01$ ), and patients with a first PFS of $>6$ months (median, 5.7 vs. 2.2 months respectively; HR, 0.44; 95\% CI, 0.20-0.96; $\mathrm{P}=0.04$ ).

The analysis of OS times revealed that the TBP group had a survival time similar to that of the non-TBP group (median, 10.8 vs. 9.5 months, respectively; HR, 1.06; 95\% CI, 0.52-2.16; $\mathrm{P}=0.88$ ). The OS time of the TBP group was also equivalent to that of the non-TBP group in the subgroups with HER2 IHC 3+ (median, 11.3 vs. 8.8 months respectively; HR, $0.97 ; 95 \% \mathrm{CI}, 0.39-2.43 ; \mathrm{P}=0.94)$, histology of intestinal type (median, 16.4 vs. 8.8 months, respectively; HR, 0.53 ; 95\% CI, 0.21-1.36, $\mathrm{P}=0.19$ ), and first PFS of $>6$ months (median, 16.3 vs. 11.5 months respectively; HR, 0.62; 95\% CI, 0.25-1.57; $\mathrm{P}=0.31$.
No statistically significant differences in the percentages of patients who received third-line chemotherapy (69.2 vs. 80.0\%, respectively; $\mathrm{P}=0.51)$, and subsequent trastuzumab-containing therapy ( 30.7 vs. $15.0 \%$, respectively; $\mathrm{P}=0.15$ ), were identified between the TBP and non-TBP groups. Fourth-line chemotherapy or beyond was administered to $7(26.9 \%)$ patients in the TBP group and 8 (40.0\%) in the non-TBP group.

Toxicity. The majority of adverse events associated with chemotherapy (Table II) were grade 1 or 2 . The frequency of any hematological toxicity of grades 3-4 was $62 \%$ in the TBP group and $50 \%$ in the non-TBP group, with no significant difference observed $(\mathrm{P}=0.43)$. The most common grade 3-4 hematological toxicity was neutropenia in the two groups: $42 \%$ in the TBP group and $40 \%$ in the non-TBP group. The frequencies of any non-hematological toxicity of grade 3-4 were also not different between the TBP and non-TBP groups ( 23 vs. $25 \%$, respectively; $\mathrm{P}=0.88$ ). No cardiac dysfunction occurred in either group.

\section{Discussion}

To the best of our knowledge, the present study is the first to evaluate a TBP strategy in second-line chemotherapy for HER2-positive AGC. There were two major findings. Firstly, the trastuzumab-combined therapy was numerically superior to chemotherapy alone for PFS, particularly in the subgroups with HER2 IHC 3+ tumors, histology of intestinal type, or a longer first PFS time ( $>6$ months). Secondly, there was no significant difference in toxicity identified between the two groups, including cardiac dysfunction. These results suggest that the TBP strategy may be a second-line treatment option for patients with HER2-positive AGC.

Compared with the results from a prospective large observational study and a randomized phase III study involving metastatic breast cancer, which showed that the continued therapy with TBP improves the survival outcome of patients with metastatic HER2-positive breast cancer $(19,20)$, the results of the present study did not demonstrate a clear 
Table II. Adverse events of all grades and grades 3-4 occurring in the non-TBP and TBP groups.

\begin{tabular}{|c|c|c|c|c|c|}
\hline \multirow[b]{2}{*}{ Adverse events } & \multicolumn{2}{|c|}{ Non-TBP $(n=20)$} & \multicolumn{2}{|c|}{ TBP $(n=26)$} & \multirow[b]{2}{*}{ P-value } \\
\hline & $\begin{array}{c}\text { All grades, } \\
\mathrm{n}(\%)\end{array}$ & $\begin{array}{c}\text { Grades 3-4, } \\
\text { n }(\%)\end{array}$ & $\begin{array}{c}\text { All grades, } \\
\text { n }(\%)\end{array}$ & $\begin{array}{c}\text { Grades } 3-4, \\
\mathrm{n}(\%)\end{array}$ & \\
\hline \multicolumn{6}{|l|}{ Hematological toxicities } \\
\hline Any & $16(80)$ & $10(50)$ & $23(88)$ & $16(62)$ & $0.43^{\mathrm{b}}$ \\
\hline Leukopenia & $12(60)$ & $5(25)$ & $18(69)$ & $7(27)$ & $0.88^{\mathrm{c}}$ \\
\hline Neutropenia & $11(55)$ & $8(40)$ & $19(73)$ & $11(42)$ & $0.87^{b}$ \\
\hline Anemia & $14(70)$ & $6(30)$ & $18(69)$ & $7(27)$ & $0.82^{\mathrm{b}}$ \\
\hline Thrombocytopenia & $4(20)$ & $1(5)$ & $1(4)$ & $0(0)$ & $0.43^{\mathrm{c}}$ \\
\hline \multicolumn{6}{|c|}{ Non-hematological toxicities } \\
\hline Any & $18(90)$ & $5(25)$ & $21(81)$ & $6(23)$ & $0.88^{c}$ \\
\hline Nausea & $11(55)$ & $2(10)$ & $7(27)$ & $2(8)$ & $1.00^{\mathrm{c}}$ \\
\hline Anorexia & $7(35)$ & $1(5)$ & $5(19)$ & $1(4)$ & $1.00^{\mathrm{c}}$ \\
\hline Fatigue & $8(40)$ & $2(10)$ & $12(46)$ & $1(4)$ & $0.57^{\mathrm{c}}$ \\
\hline Diarrhea & $6(30)$ & $2(10)$ & $3(12)$ & $0(0)$ & $0.18^{\mathrm{c}}$ \\
\hline Stomatitis & $2(10)$ & $0(0)$ & $3(12)$ & $0(0)$ & $1.00^{\mathrm{d}}$ \\
\hline Peripheral neuropathy & $7(35)$ & $0(0)$ & $11(42)$ & $1(4)$ & $1.00^{\mathrm{c}}$ \\
\hline Infusion reaction & $0(0)$ & $0(0)$ & $0(0)$ & $0(0)$ & $1.00^{\mathrm{d}}$ \\
\hline Cardiac dysfunction & $0(0)$ & $0(0)$ & $0(0)$ & $0(0)$ & $1.00^{\mathrm{d}}$ \\
\hline Febrile neutropenia & $1(5)$ & $1(5)$ & $2(8)$ & $2(8)$ & $1.00^{\mathrm{c}}$ \\
\hline
\end{tabular}

${ }^{\mathrm{a} C}$ Comparison of adverse events of grade 3 or more; ${ }^{\mathrm{b}} \chi^{2}$ tests; ${ }^{\mathrm{c} F i s h e r}$ 's exact test; ${ }^{\mathrm{d}}$ comparison of adverse events of all grades. TBP, trastuzumab beyond progression.

survival time benefit associated with trastuzumab therapy following first disease progression. However, the magnitude of unadjusted HR (0.63) for PFS in the present study was of the same order as that (0.69) in a German phase III trial comparing trastuzumab plus capecitabine vs. capecitabine alone in HER2-positive breast cancer patients whose disease had progressed during trastuzumab therapy (20), a finding which justified further investigation of HER2-positive AGC. The phenomenon of clinically relevant improvement of PFS time in the subgroup of patients with HER2 IHC 3+ tumors is congruent with the exploratory findings from the ToGA and TyTAN trials $(16,17)$. The preplanned analyses of the ToGA trial revealed the greatest survival time gain in the subgroup of patients with HER2 IHC 3+ tumors, and in the TyTAN trial, the subgroup analysis based on HER2 IHC status identified significant survival time improvement only in the IHC $3+$ subgroup.

Furthermore, the present study identified that patients with histology of intestinal-type demonstrated increased PFS time with trastuzumab treatment, which supported the subgroup result from the ToGA study in which patients with intestinal-type tumors had increased survival rates on trastuzumab-based chemotherapy (HR, 0.69; 95\% CI, 0.54-0.88). The differences in efficacy according to HER2 IHC status and histological type may be partly explained by HER 2 heterogeneity, which is more frequent in gastric cancer than in breast cancer (25). Previously, Lee et al (25) reported that only $8(32.0 \%)$ of the patients studied exhibited heterogeneous HER2 overexpression in the IHC 3+ subgroup whereas, in the IHC $2+$ subgroup, it was identified in $46(95.8 \%)$ of the 48 cases. Furthermore, only $3(14.3 \%)$ of 21 patients with diffuse or mixed types exhibited homogeneous HER 2 overexpression vs. $16(30.8 \%)$ of the 52 cases with histology of intestinal type (25). Presumably, selection of subclones lacking HER 2 overexpression is likely to confer resistance to anti-HER2 therapy in tumors with HER 2 heterogeneity, and verification of this hypothesis is required in the future. In the present study, patients who had a longer first PFS ( $>6$ months) benefited from continuing TBP, and this result is in accord with that of a previous study on metastatic breast cancer, in which patients with a longer first time-to-progression ( $\geq 8.6$ months) had significantly improved survival times following first progression compared with those who had a shorter first time-to-progression (median, 24.3 vs. 15.4 months, respectively; $\mathrm{P}=0.024$ ) (26). The dose of trastuzumab was $8 \mathrm{mg} / \mathrm{kg}$ on day 1 of the first cycle, followed by $6 \mathrm{mg} / \mathrm{kg}$ every 3 weeks, and this dose was representative of doses used by other studies (26). In the current study, it is unclear why OS time was not statistically different between the two groups, but it may be due to a high percentage of third-line or later treatment and trastuzumab use following second progression in the non-TBP group.

The limitations of this study are its retrospective nature and small sample size. In addition, the TBP strategy involves the additional cost of trastuzumab, and the current standard care in second-line treatment for gastric cancer is paclitaxel plus ramucirumab regardless of HER2 status (27); consequently, a certain degree of prudence is required when adapting this 
strategy to patients. Therefore, these findings require confirmation in a well-designed prospective study.

There was no significant difference in toxicity identified between the two groups, a finding that is consistent with the results of the ToGA trial, in which the addition of trastuzumab did not increase the toxic effects associated with standard chemotherapy (16). In addition, no cardiac dysfunction was observed in either group, in contrast to the findings from a previous study of breast cancer (28) and the ToGA trial. In the study of breast cancer, the incidence rates of cardiotoxicity were $27 \%$ for patients who received trastuzumab combined with anthracyclines and $13 \%$ for those who received paclitaxel (28). In the ToGA trial, the incidence rate of cardiac events was as low as $6 \%$ for AGC patients (16). The lack of previous treatment with anthracyclines, the short duration of exposure to trastuzumab, and underestimation of cardiac dysfunction due to less-frequent evaluation may lead to a lack of detection of cardiac events.

To summarize, although the retrospective nature of this study and the small number of patients analyzed are major limitations, these findings indicate that trastuzumab-combined therapy provided a possible benefit of improved PFS time for subgroups of AGC patients with HER2 IHC 3+ tumors, histology of intestinal type, or a longer first PFS time ( $>6$ months). An ongoing randomized phase II study (UMIN000009297; target sample size, $n=90$ ) comparing trastuzumab plus paclitaxel vs. paclitaxel-only following disease progression in patients treated with trastuzumab as first-line chemotherapy for HER2-positive AGC is likely to provide further insights into this strategy.

\section{Acknowledgements}

The authors thank Enago (www.enago.jp) for the English language review.

\section{References}

1. Ferlay J, Soerjomataram I, Dikshit R, Eser S, Mathers C Rebelo M, Parkin DM, Forman D and Bray F: Cancer incidence and mortality worldwide: Sources, methods and major patterns in GLOBOCAN 2012. Int J Cancer 136: E359-E386, 2015.

2. Glimelius B, Ekström K, Hoffman K, Graf W, Sjödén PO, Haglund U, Svensson C, Enander LK, Linné T, Sellström H and Heuman R: Randomized comparison between chemotherapy plus best supportive care with best supportive care in advanced gastric cancer. Ann Oncol 8: 163-168, 1997.

3. Murad AM, Santiago FF, Petroianu A, Rocha PR, Rodrigues MA and Rausch M: Modified therapy with 5-fluorouracil, doxorubicin, and methotrexate in advanced gastric cancer. Cancer 72: 37-41, 1993.

4. Pyrhonen S, Kuitunen T, Nyandoto P and Kouri M: Randomised comparison of fluorouracil, epidoxorubicin and methotrexate (FEMTX) plus supportive care with supportive care alone in patients with non-resectable gastric cancer. Br J Cancer 71: 587-591, 1995.

5. Ajani JA, Moiseyenko VM, Tjulandin S, Majlis A, Constenla M, Boni C, Rodrigues A, Fodor M, Chao Y, Voznyi E, et al: Clinical benefit with docetaxel plus fluorouracil and cisplatin compared with cisplatin and fluorouracil in a phase III trial of advanced gastric or gastroesophageal cancer adenocarcinoma: The V-325 Study Group. J Clin Oncol 25: 3205-3209, 2007.

6. Cunningham D, Starling $\mathrm{N}$, Rao S, Iveson $\mathrm{T}$, Nicolson $\mathrm{M}$, Coxon F, Middleton G, Daniel F, Oates J and Norman AR; Upper Gastrointestinal Clinical Studies Group of the National Cancer Research Institute of the United Kingdom: Capecitabine and oxaliplatin for advanced esophagogastric cancer. N Engl J Med 358: 36-46, 2008.
7. Koizumi W, Narahara H, Hara T, Takagane A, Akiya T, Takagi M, Miyashita K, Nishizaki T, Kobayashi O, Takiyama W, et al: S-1 plus cisplatin versus S-1 alone for first-line treatment of advanced gastric cancer (SPIRITS trial): A phase III trial. Lancet Oncol 9: 215-221, 2008

8. Ford HE, Marshall A, Bridgewater JA, Janowitz T, Coxon FY, Wadsley J, Mansoor W, Fyfe D, Madhusudan S, Middleton GW, et al: Docetaxel versus active symptom control for refractory oesophagogastric adenocarcinoma (COUGAR-02): An open-label, phase 3 randomised controlled trial. Lancet Oncol 15: 78-86, 2014.

9. Hironaka S, Ueda S, Yasui H, Nishina T, Tsuda M, Tsumura T, Sugimoto N, Shimodaira H, Tokunaga S, Moriwaki T, et al: Randomized, open-label, phase III study comparing irinotecan with paclitaxel in patients with advanced gastric cancer without severe peritoneal metastasis after failure of prior combination chemotherapy using fluoropyrimidine plus platinum: WJOG 4007 trial. J Clin Oncol 31: 4438-4444, 2013.

10. Kang JH, Lee SI, Lim DH, Park KW, Oh SY, Kwon HC, Hwang IG, Lee SC, Nam E, Shin DB, et al: Salvage chemotherapy for pretreated gastric cancer: A randomized phase III trial comparing chemotherapy plus best supportive care with best supportive care alone. J Clin Oncol 30: 1513-1518, 2012.

11. Kim HS, Kim HJ, Kim SY, Kim TY, Lee KW, Baek SK, Kim TY, Ryu MH, Nam BH and Zang DY: Second-line chemotherapy versus supportive cancer treatment in advanced gastric cancer: A meta-analysis. Ann Oncol 24: 2850-2854, 2013.

12. Aizawa M, Nagatsuma AK, Kitada K, Kuwata T, Fujii S, Kinoshita T and Ochiai A: Evaluation of HER2-based biology in 1,006 cases of gastric cancer in a Japanese population. Gastric Cancer 17: 34-42, 2014.

13. Barros-Silva JD, Leitão D, Afonso L, Vieira J, Dinis-Ribeiro M, Fragoso M, Bento MJ, Santos L, Ferreira P, Rêgo S, et al: Association of ERBB2 gene status with histopathological parameters and disease-specific survival in gastric carcinoma patients. Br J Cancer 100: 487-493, 2009.

14. Takehana T, Kunitomo K, Kono K, Kitahara F, Iizuka H, Matsumoto Y, Fujino MA and Ooi A: Status of c-erbB-2 in gastric adenocarcinoma: A comparative study of immunohistochemistry, fluorescence in situ hybridization and enzyme-linked immuno-sorbent assay. Int J Cancer 98: 833-837, 2002.

15. Yano T, Doi T, Ohtsu A, Boku N, Hashizume K, Nakanishi M and Ochiai A: Comparison of HER2 gene amplification assessed by fluorescence in situ hybridization and HER 2 protein expression assessed by immunohistochemistry in gastric cancer. Oncol Rep 15: 65-71, 2006.

16. Bang YJ, Van Cutsem E, Feyereislova A, Chung HC, Shen L, Sawaki A, Lordick F, Ohtsu A, Omuro Y, Satoh T, et al: Trastuzumab in combination with chemotherapy versus chemotherapy alone for treatment of HER2-positive advanced gastric or gastro-oesophageal junction cancer (ToGA): A phase 3 , open-label, randomised controlled trial. Lancet 376: 687-697, 2010.

17. Satoh T, Xu RH, Chung HC, Sun GP, Doi T, Xu JM, Tsuji A, Omuro Y, Li J, Wang JW, et al: Lapatinib plus paclitaxel versus paclitaxel alone in the second-line treatment of HER2-amplified advanced gastric cancer in asian populations: TyTAN-a randomized, phase III study. J Clin Oncol 32: 2039-2049, 2014.

18. Dai GH, Shi Y, Chen L, Lv YL and Zhong M: Trastuzumab combined with docetaxel-based regimens in previously treated metastatic gastric cancer patients with HER2 over-expression. Hepatogastroenterology 59: 2439-2444, 2012.

19. Jackisch C, Welslau M, Schoenegg W, Selbach J, Harich HD, Schröder J, Schmidt M, Göhler T, Eustermann H, Ringel R and Hinke A: Impact of trastuzumab treatment beyond disease progression for advanced/metastatic breast cancer on survival - results from a prospective, observational study in Germany. Breast 23: 603-608, 2014.

20. von Minckwitz G, du Bois A, Schmidt M, Maass N, Cufer T, de Jongh FE, Maartense E, Zielinski C, Kaufmann M, Bauer W, et al: Trastuzumab beyond progression in human epidermal growth factor receptor 2-positive advanced breast cancer: A German breast group 26/breast international group 03-05 study. J Clin Oncol 27: 1999-2006, 2009.

21. Oken MM, Creech RH, Tormey DC, Horton J, Davis TE, McFadden ET and Carbone PP: Toxicity and response criteria of the Eastern Cooperative Oncology Group. Am J Clin Oncol 5: 649-655, 1982.

22. NCCN Clinical Practice Guidelines in Oncology GC, Ver3. 2016. http://www.nccn.org/professionals/physician_gls/f_guidelines.asp. Accessed October 30, 2016. 
23. Nishino M, Jagannathan JP, Ramaiya NH and Van den Abbeele AD: Revised RECIST guideline version 1.1: What oncologists want to know and what radiologists need toknow. AJR AmJ Roentgenol 195: 281-289, 2010.

24. National Cancer Institute Common Terminology Criteria for Adverse Events (CTCAE) version 4.0. https://evs.nci.nih. gov/ftpl/CTCAE/About.html.

25. Lee HE, Park KU, Yoo SB, Nam SK, Park do J, Kim HH and Lee HS: Clinical significance of intratumoral HER2 heterogeneity in gastric cancer. Eur J Cancer 49: 1448-1457, 2013.

26. Hayashi M, Okumura Y, Osako T, Toyozumi Y, Arima N, Iwase H and Nishimura R: Time to first tumor progression as a predictor of efficacy of continued treatment with trastuzumab beyon progression in human epidermal growth factor receptor 2-positive metastatic breast cancer. Int J Clin Oncol 16: 694-700, 2011.
27. Wilke H, Muro K, Van Cutsem E, Oh SC, Bodoky G, Shimada Y, Hironaka S, Sugimoto N, Lipatov O, Kim TY, et al: Ramucirumab plus paclitaxel versus placebo plus paclitaxel in patients with previously treated advanced gastric or gastro-oesophageal junction adenocarcinoma (RAINBOW): A double-blind, randomised phase 3 trial. Lancet Oncol 15: 1224-1235, 2014.

28. Seidman A, Hudis C, Pierri MK, Shak S, Paton V, Ashby M, Murphy M, Stewart SJ and Keefe D: Cardiac dysfunction in the trastuzumab clinical trials experience. J Clin Oncol 20: 1215-1221, 2002. 